\title{
An Ssvep-Based Bci System and its Applications
}

\author{
Jzau-Sheng Lin \\ Dept. of Computer Science and Information Eng., \\ National Chin-Yi University of Technology \\ No.57, Sec. 2, Zhongshan Rd., Taiping Dist., \\ Taichung 41170, Taiwan
}

\author{
Cheng-Hung Shieh \\ Dept. of Computer Science and Information Eng., \\ National Chin-Yi University of Technology \\ No.57, Sec. 2, Zhongshan Rd., Taiping Dist., \\ Taichung 41170, Taiwan
}

\begin{abstract}
A Brain-Computer-Interface (BCI) based system with a System on a Programmable Chip (SOPC) platform by using of the Steady-State Visually Evoked Potentials (SSVEP) through a Bluetooth interface was proposed in this paper. The proposed BCI system can aid the Amyotrophic Lateral Sclerosis (ALS) or other paralyzed patients to easily control an electric wheelchair in their live. The electroencephalogram (EEG) signals may be detected by electrodes and extracting chip when the patients gazed a flickered target with a specific frequencies. Then these signals can be transformed by FFT into frequency domain and then transmitted to the hardware of electric wheelchair by using of Bluetooth interface. Finally, the electric wheelchair can be moved smoothly in accordance with commands converted by the frequencies of the EEG signal. The experimental results had shown that the proposed system can easily control electric wheelchairs.
\end{abstract}

Keywords-Brain-Computer-Interface $\quad($ BCI $) ; \quad$ Steady-State
Visually Evoked Potentials (SSVEP); Electroencephalogram
(EEG)

\section{INTRODUCTION}

People's behavior and activities will be controlled by signals in the brain. The signals are then delivered to the entire body via the nervous system. Some people can't control hands and body but their brains are still operating like a normal person such as amyotrophic lateral sclerosis (ALS), muscular dystrophy, and severe cerebral palsy that is also referred to as motor neuron disease. The electric wheelchair has been considered as one of important mobility aids for the elderly as well as the physically impaired patients. Including paralyzed patients, approximately $50 \%$ of patients cannot be able to control an electric wheelchair by conventional methods in the clinicians report. Especially, people can only use eyes and brain to exercise their willpower if they got motor neuron disease motor neuron disease (MND).

In the research of signal transformation in brain science, BCI system is created to obtain the human EEG signals in order to build an interactive system, and converted them into commands that enable advanced algorithms, or computer system to identify and deal with these commands. The BCI provides a communication channel that allows the user by the strength of brain wave signals to communicate with the outside world through the brain activity to directly infer the subject's intention to transform into a computer-controlled signal. It can provide patients who suffer from motor neuron disease a new auxiliary interface and can also allow physically disabled patients to have basic self-control environment and making them look more dignified in their life. BCI is a system so that people can directly communicate with the external device through the neuromuscular pathway in references [1-3]. In some researches, BCI system is a promising tool which can help the paralyzed people such as medical assistant devices. A BCI system may contain acquisition of EEG signal, signal processing, and application interfaces. The signal processing includes preprocessing, feature extraction, and classification.

In the past two decades, different EEG signal characteristics such as mu / beta rhythm, the P300 eventrelated potentials and visual evoked potential (VEP) has been widely used in the field of BCI. The VEP system has its advantages including higher information transfer rate (ITR), a small amount of training samples, low users' variable, and easy use.

SSVEP signals that are natural responses to visual stimulation at particular frequencies ranging from $3.5 \mathrm{~Hz}$ to 75 $\mathrm{Hz}$ [4-6]. When the eyes are excited by a visual stimulus signal and the brain then generates same reaction at the same frequency of the visual stimulation signal. The characteristic of SSVEP is that it can detect and measure SSVEP stimulation frequencies when the amplitude of the stimulation frequency is increased. Frequency coding method has been widely used in the SSVEP-based BCI systems. In such a system, visual targets are flickering with different frequencies. The system can identify the primary frequency of SSVEP when the subject gazes a target. To design a practical BCI system needs to address several issues such as ease of use, a reliable system performance, and low-cost hardware and software. In recent years, with the biomedical sciences and electronics technology, mobile and online BCI's development has been proposed. SSVEP has been widely used in EEG visual research as a task, because it does not require special training. It also has a very high information transmission rate (ITR).

For the proposed electric wheelchairs [7-10], they did not use any wireless interface in their system. The authors also proposed EEG-based electric wheelchairs with microprocessorbased and FPGA-based through wireless interface [11-12]. Although they can simplifies and downsizes the system with wireless and FPGA manner, the speed of electric wheelchair was limited since the "attention" signal attracted from forehead can just converted one command. In order to speed up the movement for an electric wheelchair and code several commands, an SSVEP technique was developed to extract brain signals on the occipital in this paper.

This paper is organized as follows. The main system architecture and subsystems' structure are introduced in 
Section 2. Section 3 shows the experimental results. Finally, Section 4 is the discussion and conclusions.

\section{SySTEM ARCHITECTURE}

The proposed SSVEP-based BCI system with an SOPC platform through Bluetooth interface for electric wheelchair is shown in Fig. 1. The architecture includes a stimulating platform, EEG signal acquisition unit, signal processing unit, and electric wheelchair with an SOPC platform.

In the applications of SSVEP-based BCI system, several papers [13-15] indicated that the low frequency region has stronger amplitude response. They proposed SSVEP-based BCI systems with the low-frequency region because these systems occupy a high accuracy rate. For example, Cecotti13 developed a Calibration-Less SSVEP-based BCI spelling system using the frequency band between 6.67 and $8.57 \mathrm{~Hz}$ for the commands. Ortner et al [14]. proposed a hand orthosis control system by using of an SSVEP-based BCI with two commands which flickered between 8 and $13 \mathrm{~Hz}$ built on the orthosis. Hwang [15] et al. demonstrated a speller with SSVEP-based BCI system between 5 and $9.9 \mathrm{~Hz}$. In the proposed stimulating platform, three arrows, indicating moving ahead, turning left, and turning right for electric wheelchair, were flickered with different low frequencies such as $9 \mathrm{~Hz}$, $11 \mathrm{~Hz}$, and $13 \mathrm{~Hz}$ on the screen of ASUS PadFone in order to get higher accuracy rate. Then, a patient gazes target arrow to generate the correspondence frequency on his/her occipital. In this system, electrodes are attached on point FP2 of forehead for eyes winking and point $\mathrm{Oz}$ of occipital for stimulated frequencies. In the EEG acquisition unit, the EEG or eye winking signal was extracted and processed by NeuroSky EEG chips. Then, these signals were transmitted through a Bluetooth transmitter to a Bluetooth receiver in the signal processing unit on the platform of ASUS PadFone. In addition to the Bluetooth receiver, the signal processing unit occupied FFT module to transform EEG signal from spatial domain to frequency domain for recognizing frequencies. It also detects an eye winking signal on point FP2 with a peak pulse on spatial domain. When a suitable frequency or eye-winking pulse was detected, they would be transformed into a correspondence commend and transmitted to the SOPC platform on the electric wheelchair through another Bluetooth interface. In the electric wheelchair with an SOPC platform, a Bluetooth receiver to receiver a command and 4-set ultrasound modules to detect obstacles are mounted. These ultrasound modules were mounted through Universal Asynchronous Receiver/ Transmitter (UART) interface. Then, these signals can be converted to amplitudes of voltage by a Digital to Analog Converter (DAC) and transmitted by a General Purpose Input Output (GPIO) interface on the SOPC to control DC motors on the electric wheelchair.

\section{A. EEG Acquisition Unit}

Fig. 2 shows the EEG acquisition unit. The brain wave is extracted by using of an acquisition chip produced by NeuroSky corporation and named TGAM1. The Bluetooth wireless module HL-MR08R-C2A serves as a data transmitter. HL-MR08R-C2A was selected because it has low-power consumption, supports many interface protocols (SPP, SDP, GAP, L2CAP, and RFCOMM), and can be designed a wireless interface with a simple manner. In order to effectively acquire the suitable EEG signals, 2-channel electrodes were bounded on the point FP2 of forehead, point $\mathrm{Oz}$ of occipital, and grounding electrode was tied on one's ears. The International 10-20 system is a reference to apply the locations of scalp electrodes for EEG extraction. The proposed system uses a set of channel for acquiring the brainwave signals. We used FP2 and $\mathrm{Oz}$ as the points of interception. $\mathrm{A} 1$ and $\mathrm{A} 2$ on the earlobes are set as the EEG reference points. The wet electrodes are placed on the occipital lobe of the scalp as shown as in Fig. 3.

In the proposed platform, two NeuroSky EEG chips that work in voltage $3.3 \mathrm{~V}$ with 57600 transmission baud rate. The EEG are classified into five types in the TGAM1 including Signal quality, Attention, Meditation, Raw EEG, and Long EEG. The size of Raw EEG is 2 bytes. The size of Long EEG is 24 bytes including Delta, Theta, Low Alpha, High Alpha, Low Beta, High Beta, Low Gamma, and Mid Gamma. The Bluetooth module named HL-MD08R-C2A is also embedded for the wireless interface. In this paper, Raw EEG was used for detecting different frequencies from occipital lobe and extracted eyes-winking signal from forehead. It also uses two Bluetooth transmitters in the EEG acquisition device to transmit signals extracted from locations $\mathrm{Oz}$ and FP2. The transferred rate is $3 \mathrm{Mbit} / \mathrm{s}$ as well as the data transferred band is $2.4 \mathrm{GHz}$. The operating voltage is $3.3 \mathrm{~V}$. In this paper the ASUS PadFone was also selected for the development platform.

The hardware diagram of EEG Acquisition Unit is shown as in Fig. 4. In the NeuroSky TGAM1, EEG signals are extracted from EEG electrodes and sent from TXD to RX in Bluetooth module. Finally, these EEG signals are transmitted from TX to Signal Processing Unit with Bluetooth interface.

\section{B. Signal Processing Unit}

In this paper, the EEG signals were transformed by the Fast Fourier Transform (FFT) from spatial domain into frequency domain. The FFT and its inverse manner are defined as Eqs. (1) and (2), in which the signals are transformed between spatial domain and frequency domain.

$$
\begin{aligned}
& X(k)=\sum_{n=0}^{N-1} x(n) e^{j 2 n \pi k / N} \\
& x(n)=\sum_{k=0}^{N-1} X(k) e^{-j 2 n \pi k / N}
\end{aligned}
$$

FFT is an efficient processing approach to compute the discrete Fourier Transform (DFT) of a digital signal to convert spatial signal into frequency domain. FFT reduces the number of computations needed for $\mathrm{N}$ points from $2 N^{2}$ to $2 \mathrm{~N} \log _{2} N$. Therefore, EEG signal extracted from location $\mathrm{Oz}$ within 1 second in spatial domain. We obtained $\mathrm{N}=512$-point amplitudes during a second with a $512 \mathrm{~Hz}$ sampling frequency.

\section{Signal Processing Unit}

In the electric wheelchair with SOPC Platform, the commands were received from the Bluetooth interface and transmitted to the SOPC platform through an UART interface. 
The signals, detected by the 4-set ultrasound modules to detect obstacles at the fronts of left, right, left front, and right, were also sent to the SOPC module by an UART.

We utilize a 32-bit Redundancy Instruct Set Computer (RISC) SOPC platform, built in XILINX Virtex-4 XC4VFX12-FF668-10, as a processing unit for commands and ultrasound signals. These signals were implemented by using of $C$ language in order to transform the commands and obstacle signals into 4-set D/A converters by a GPIO interface. The 4set D/A converters convert digital signals, transmitted by GPIO, to analog voltages to drive DC motors on the electric wheelchair. The Virtex-4 FPGA occupies 64-MB DDR SDRAM, 32-bit interface running up to $266-\mathrm{MHz}$ data rate. It uses eight independent I/O banks to support 32 different singleended and differential I/O standards and allows us easily to migrate different densities across multiple packages. The Virtex-4 SOPC platform integrates many Silicon Intellectual Property (SIP) modules, including RS-232, RJ-45, USB, expand I/O pin, etc. The processing of commands and ultrasound signals can be developed by the Xilinx Embedded Development Kit (EDK), in which the Platform Studio (XPS) and IP cores (including a 32-bit soft-RISC-CPU MicroBlaze) are supported.

Fig. 5 shows the hardware diagram of the Bluetooth receiver in the electric wheelchair with SOPC platform and power supplies from $25 \mathrm{~V}$ battery to $5 \mathrm{~V}$ and $15 \mathrm{~V}$, respectively. A Bluetooth module is set in order to receive commands from Signal Processing Unit. Then, these commands are sent to Virtex-4 from ports TX through an UART.

The 4-set D/A converters, were used to generate different voltages to mount on the connecter to replace the joystick module of the VR2 wheelchair control system (PG Drivers Technology). The hardware diagram of 4-set DACs, constructed by four D/A converters named DAC0830, are shown as in Fig. 6. The SOPC sent four bytes data to the four DACs to generate four analog signals. These four analog signals are then sent to the VR2 control system. The electric wheelchair, control by VR2, is shown as in Fig. 7.

\section{EXPERIMENTAL RESULTS}

The experimental environment is also to refer the scenario in reference [11]. The length of travel path for the electric wheelchair from start point and bypassing two tables then going back to the original point is about 24 meters. We also selected seven healthy young people. Everyone must test 3 times. In reference [11], the EEG signals, attention and eye winking, was just extracted on electrode position FP2. In order to downsizing the size of hardware, the FPGA scheme was used to implement the control system in electric wheelchair and proposed in reference [12]. Owing to just using attention and eye winking signals in references [11] and [12], the speed of electric wheelchair was limited. Therefore, an SSVEP-based $\mathrm{BCI}$ system is proposed in this paper in order to extend control commands and simplify the learning process for the patients. The experimental results for the consuming time are shown as in Table 1. From Table 1, we can find that the average consume time of the proposed SSVEP-based electric wheelchair (03:34) and attention-based in the reference [11] $(07: 14)$. It proved that the proposed system is faster than the system in references [11] and [12] over two times. The maximum consuming time for the proposed system were less than 5 minutes and 20 seconds while the maximum consuming time is 12 minutes and 52 seconds for the references [11] and [12]. From the experimental results, promising results can be obtained by the proposed electric wheelchair.

The Information Transmission Rate (ITR) is generally used to estimate the performance of the communication and control for brain-computer interfaces [16]. The higher value indicates the more performance. The proposed method is also used ITR to assess the performance of the system. The ITR is defined by

$$
\begin{aligned}
& \frac{\text { Bits }}{\text { Command }}=\log _{2} N+P \log _{2} P+(1-P) \log _{2} \frac{1-P}{N-1} \\
& I T R=\frac{\text { Bits }}{\text { Command }} \cdot \frac{60}{C T I}
\end{aligned}
$$

where $N$ is the total number of commands ( $N=4$ in our system), $P$ is the probability of correct selection, and $C T I$, expressed as Command Transfer Interval, is the average time during a second for one command.

In this paper, the time, shown as in Table 2, is the decision time of commands, which was calculated by the total consumed time minus running time of the wheelchair. For example, the subject 1 consumed 3 minutes and 38 seconds to complete an experiment, in which he wasted about 50 seconds to decide the wheelchair going ahead, turning right or turning left with $\mathrm{CTI}=1.92$ and ITR $=62.4$ in Test 1 . The average decision time of commands with completing an experiment is 51.6 seconds for 7 subjects. And, the average CTI and ITR are 1.98 and 60.78 , respectively.

\section{DisCUSSION AND CONCLUSIONS}

In this paper, an SSVEP-based EEG signal on the occipital lobe $\mathrm{Oz}$ and eye-winking signal on the forehead FP2 through a BCI interface for electric wheelchair with wireless scheme was proposed. In the proposed system, a patient just gazes a flickering target with low frequencies to select different directions to force an electric wheelchair move ahead, turn left or right. The eye-winking detected from FP2 was used to enforce wheelchair stop. 4-set ultrasound modules were to detect the obstacles around the wheelchair. From the approval of experimental results, the proposed SSVEP-based electric wheelchair is low cost and easier controlled by the patients. In the process of experiments, we can find when the flickering frequencies of arrow marks is lower, the good detection effect of frequencies was obtained. But, the subjects' eye are fatigued. The flickering frequency is slightly higher that can relieve eye fatigue, but poor detection rate can be got. In the future, we can replace the frequency-based SSVEP system with phase-based SSVEP to relieve eye fatigue and to update the performance of frequency detection.

\section{ACKNOWLEDGMENT}

In this paper, the research was sponsored by the National Science Council of Taiwan under the Grants NSC102-2221-E167-032 and NSC103-2221-E-167 -027 - . 


\section{REFERENCES}

[1] F. Cabrera, O. F. do Nascimento, D. Farina, K. Dremstrup, "Braincomputer interfacing: how to control computers with thoughts," in Proc. 1st International Symposium on Applied Sciences on Biomedical and Communication Technologies: pp. 1-4, 2008.

[2] J. R. Wolpae, N. Birbaumer, D. J. McFarland, G. Pfurtscheller, and T. M. Vaughan, "Brain-computer interfaces for communication and control,” Clin Neurophsiol, vol. 113, pp. 767-791, 2002.

[3] G. E. Fabiani, D. J. McFarland, J. R. Wolpaw, and G. Pfurtscheller, "Conversion of EEG activity into cursor movement by a brain-computer interface (BCI)," IEEE Trans on Neural Systems and Rehabilitation Eng., vol. 12, pp. 331-338, 2004.

[4] Nawrocka and K. Holewa, "Brain-computer interface based on steady state visual evoked potentials (SSVEP)," In Proc. 14th International Carpathian Control Conference, pp. 251-254, 2013.

[5] L. Bi, Y. Li, K. Jie, and X. Fan, "A new SSVEP brain-computer interface based on a head up display," in Proc. Int Conference on Complex Medical Engineering, pp. 201-204, 2013.

[6] S. P. Kelly, E. C. Lalor, R. B. Reilly, and J. J. Foxe, "Visual spatial attention tracking using high-density SSVEP data for independent braincomputer communication," IEEE Trans on Neural Systems and Rehabilitation Eng., vol. 13 pp. 172-178, 2005.

[7] L. Montesano, M. Diaz, S. Bhaskar, and J. Minguez "Towards an intelligent wheelchair system for users with cerebral palsy users," IEEE Trans. on Neural Systems and Rehabilitation Eng. 18: 193-202, 2010.

[8] K. Tanaka, K. Matsunaga, and H. O. Wang, "Electroencephalogrambased control of an electric wheelchair," IEEE Trans. on Robotics, 21: 762-766, 2005.
[9] F. Gal'an, M. Nuttin, E. Lew, P. W. Ferrez, G. Vanacker, J. Philips, J. d R. Mill'an, "A brain-actuated wheelchair: asynchronous and noninvasive brain-computer interfaces for continuous control of robots, "Clinical Neurophysiology, 119: 2159-2169, 2008.

[10] S.-Y. Cho, A. P. Vinod, and K. W. E. Cheng, "Towards a braincomputer interface based control for next generation electric wheelchairs," in Proc. Int. Conf. on Power Electronics Systems and Applications, pp. 1-5, 2009

[11] J.-S. Lin, and W.-C. Yang, "Wireless brain-computer interface for electric wheelchairs with eeg and eye-blinking signals," Int. J. of Innovative Computing, Information and Control,vol 4, pp. 2973-2980, 2012.

[12] J.-S. Lin, and S.-M. Huang, "An FPGA-based brain-computer interface for wireless electric wheelchairs," Applied Mechanics and Materials, vol. 284-287, pp. 1616-1621, 2013.

[13] H, Cecotti, "A self-paced and calibration-less SSVEP-based braincomputer interface speller," IEEE Trans Neural System Rehab Eng., vol. 18, pp. 127-134, 2010.

[14] R. Ortner, B. Allison, G. Korisek, H. Gaggl, and G. Pfurtscheller, "An SSVEP BCI to control a hand orthosis for persons with tetraplegia," IEEE Trans Neural System Rehabil. Eng., vol. 19, pp. 1-5, 2011.

[15] H.-J. Hwang, J.-H. Lim, Y.-J. Jung, H. Choi, S.-W. Lee, and C.-H. Im, "Development of an SSVEP-based BCI spelling system adopting a QWERTY-style LED keyboard," Neuroscience Methods, vol. 208, pp. 59-65, 2012.

[16] J. R. Wolpaw, N. Birbaumer, D. J. Mc Farland, G. Pfurtscheller, and T. M. "Vaughan, Brain-computer interfaces for communication and control, Clinical Neurophysiology," vol. 113, pp. 67-79, 2002.
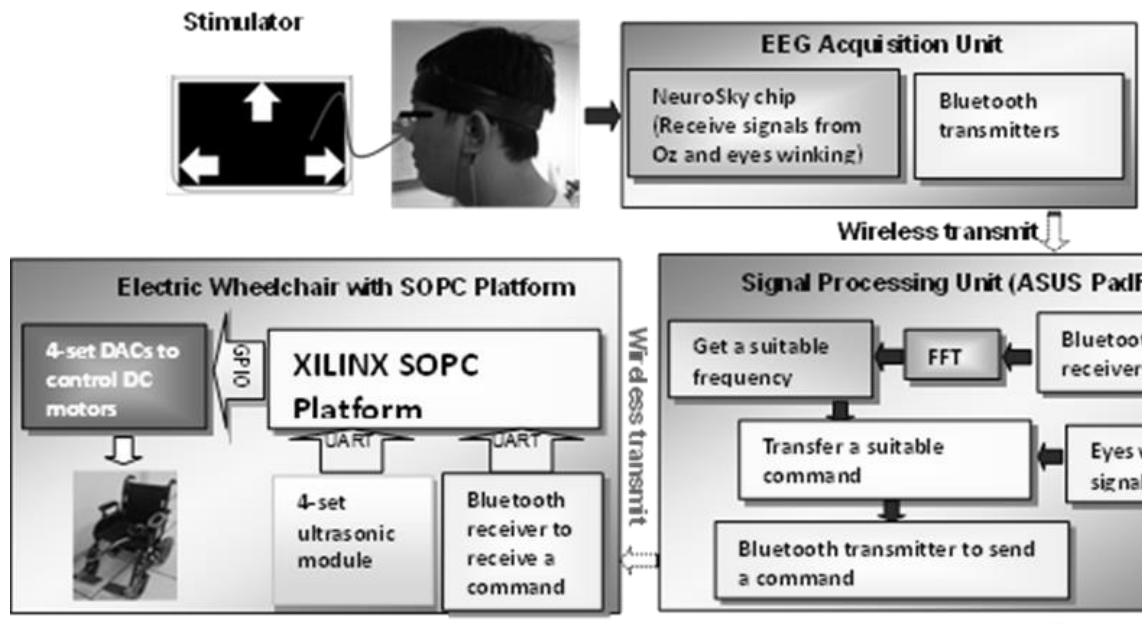

Wireless transmit

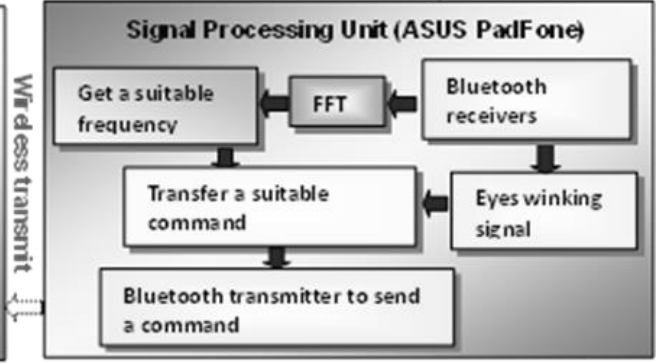

Fig. 1. System architecture of an SVEP-based electric wheelchair
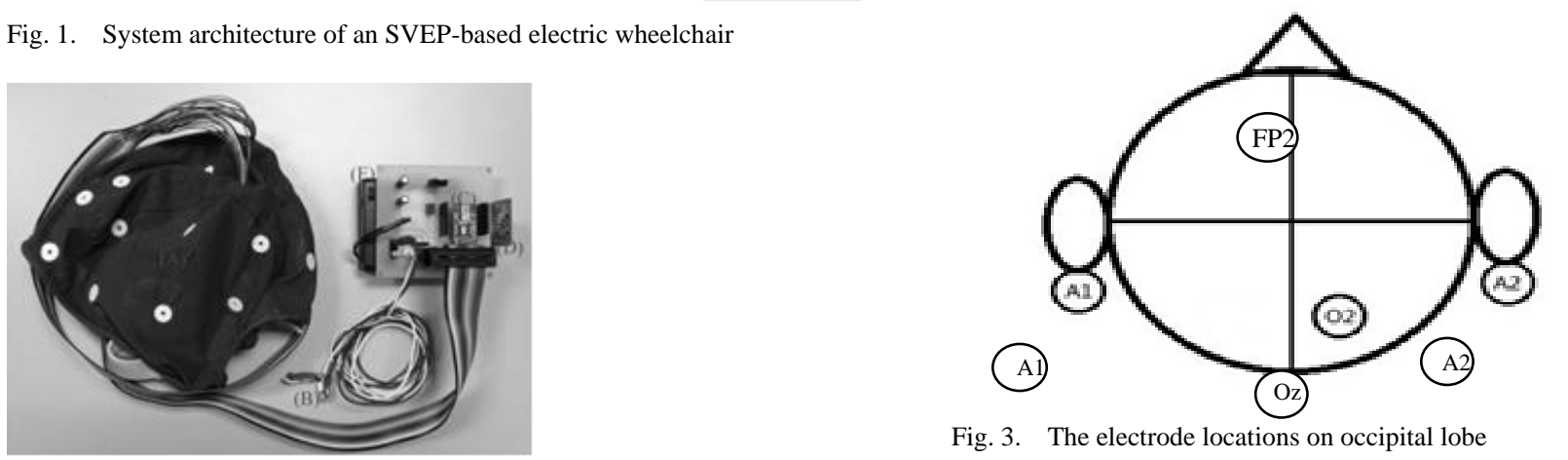

Fig. 2. The EEG acquisition device. (A)EEG caps; (B) Electrode pads; (C) NeuroSky EEG chip; (D) Bluetooth module; and (E)power supply

Fig. 3. The electrode locations on occipital lobe 


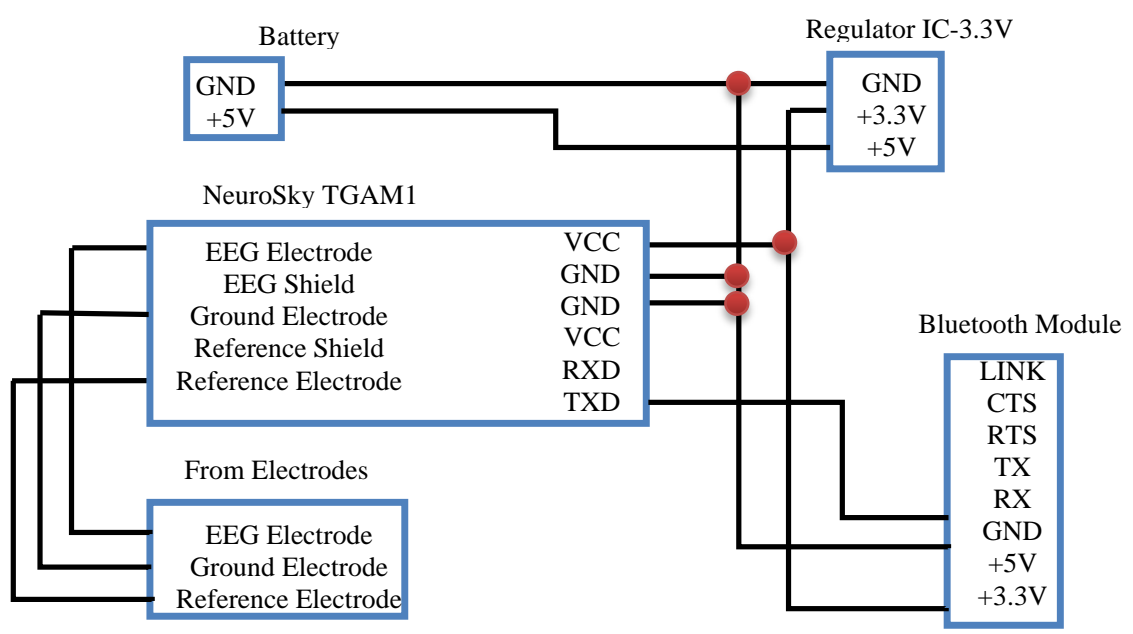

Fig. 4. Hardware diagram of the acquisition module

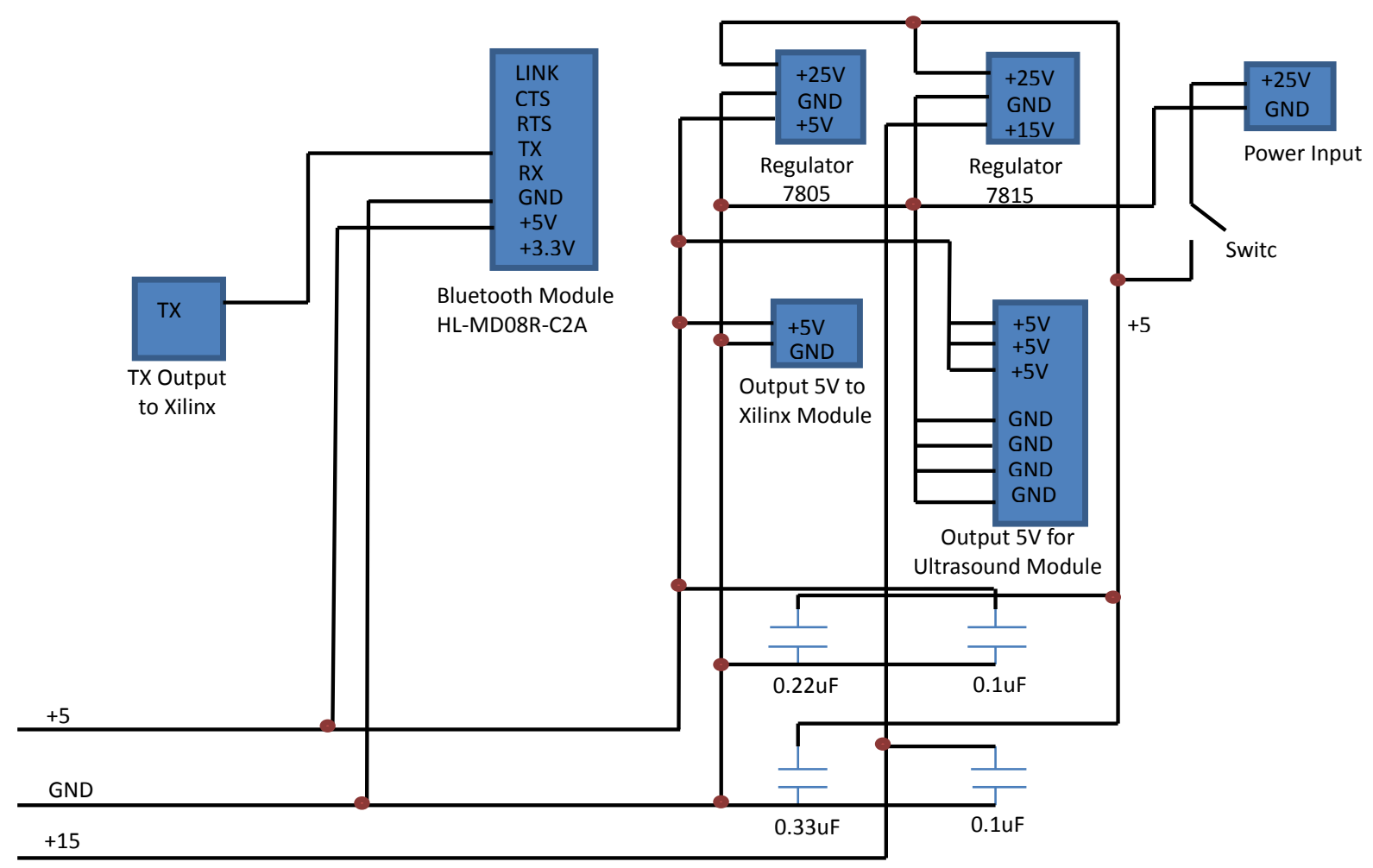

Fig. 5. Bluetooth modules with power generators 


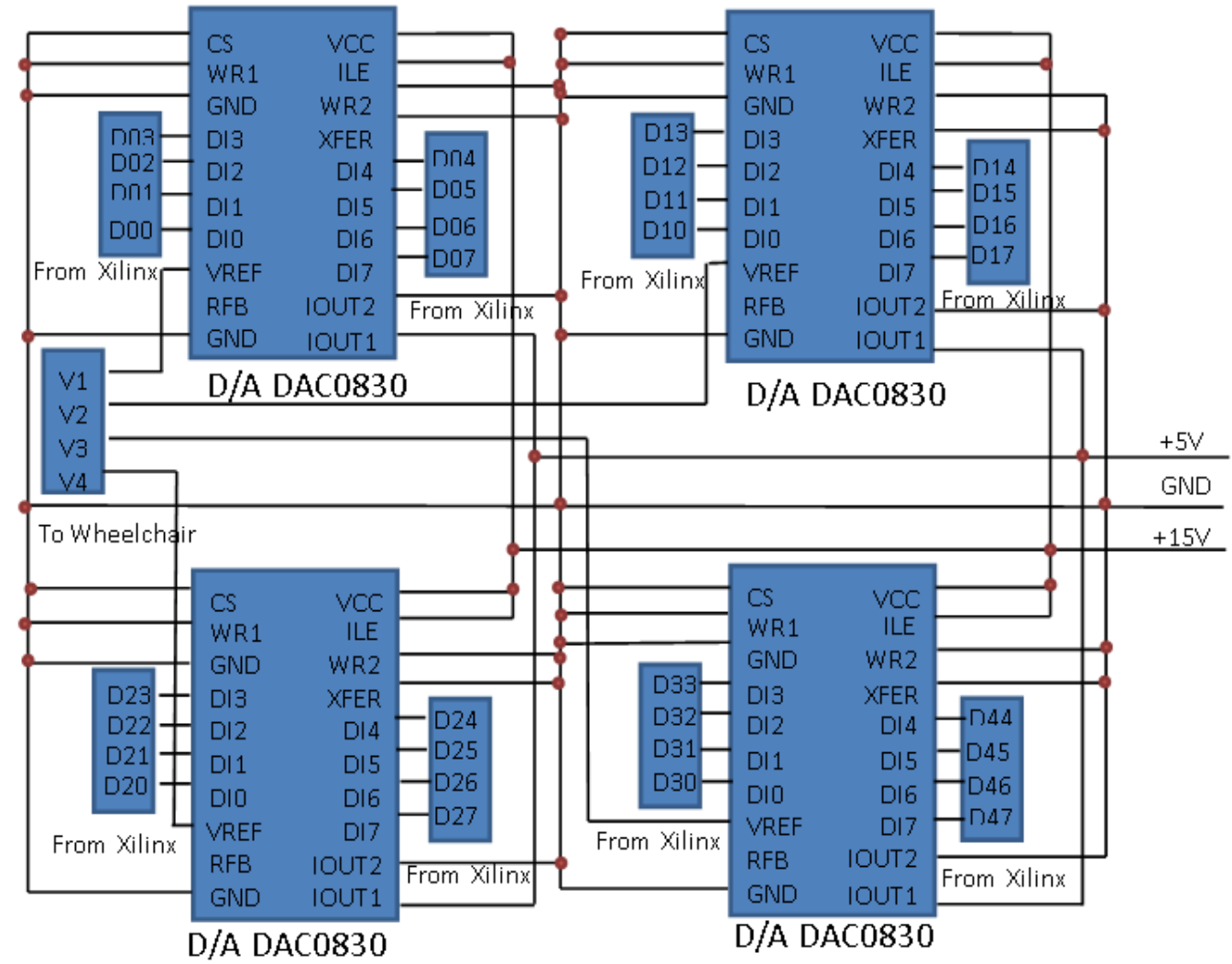

Fig. 6. Hardware diagram of 4-set DACs

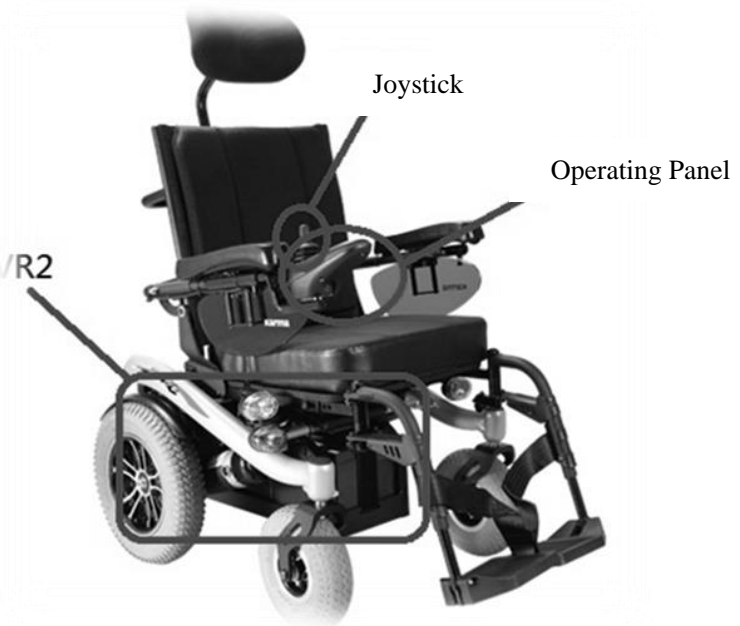

Fig. 7. VR2-controlled electric wheelchair 
TABLE I. CONSUMING TIME AND SYSTEM PERFormANCE FOR DifFERENT SUBJECTS

\begin{tabular}{|c|c|c|c|c|c|c|c|c|}
\hline \multirow{2}{*}{ Subject \# } & \multicolumn{2}{|c|}{ Test 1} & \multicolumn{2}{|c|}{ Test 2} & \multicolumn{2}{|c|}{ Test 3} & \multicolumn{2}{|c|}{ Average time } \\
\hline & Ref.11 & Proposed & Ref.11 & Proposed & Ref.11 P & Proposed & Ref.11 & Proposed \\
\hline 1 & 04.08 & 03:38 & $03: 48$ & 03:07 & $04: 15$ & $5 \quad 03: 32$ & 04:04 & 03:26 \\
\hline 2 & 07:07 & 03:06 & 07:11 & 03:09 & $05: 47$ & 03:07 & $06: 42$ & 03:07 \\
\hline 3 & $07: 59$ & $02: 58$ & $06: 31$ & $02: 50$ & $06: 42$ & 03:00 & 07:04 & 03:00 \\
\hline 4 & $04: 46$ & $03: 39$ & $03: 42$ & 03:00 & $05: 16$ & $03: 12$ & $04: 35$ & $03: 12$ \\
\hline 5 & $07: 24$ & $05: 20$ & $06: 56$ & $05: 12$ & $07: 45$ & 05:09 & $07: 22$ & 05:09 \\
\hline 6 & $08: 48$ & $03: 57$ & $07: 28$ & $03: 48$ & $10: 25$ & $03: 57$ & $08: 54$ & $03: 57$ \\
\hline 7 & $10: 34$ & $03: 20$ & $12: 52$ & $02: 58$ & $12: 22$ & 03:06 & $11: 56$ & 03:06 \\
\hline Average time & & & & & & & 07:14 & 03:34 \\
\hline
\end{tabular}

Consuming time $=$ Min:Sec

TABLE II. SYSTEM PERFORMANCE

\begin{tabular}{|c|c|c|c|c|c|c|c|c|c|c|c|c|}
\hline \multirow{2}{*}{ Subject \# } & \multicolumn{3}{|c|}{ Test 1} & \multicolumn{3}{|c|}{ Test 2} & \multicolumn{3}{|c|}{ Test 3} & \multicolumn{3}{|c|}{ Average } \\
\hline & Time & $C T I$ & $I T R$ & Time & $C T I$ & $I T R$ & Time & $C T I$ & $I T R$ & Time & $C T I$ & $I T R$ \\
\hline 1 & 50 & 1.92 & 62.40 & 51 & 1.96 & 61.18 & 51 & 1.96 & 61.18 & 51.6 & 1.95 & 61.58 \\
\hline 2 & 47 & 1.81 & 66.38 & 50 & 1.92 & 62.40 & 51 & 1.96 & 61.18 & 49.3 & 1.90 & 63.32 \\
\hline 3 & 53 & 2.04 & 58.87 & 53 & 2.04 & 58.87 & 50 & 1.92 & 62.40 & 52.0 & 2.00 & 60.05 \\
\hline 4 & 48 & 1.85 & 65.00 & 57 & 2.19 & 54.74 & 49 & 1.88 & 63.67 & 51.3 & 1.97 & 61.14 \\
\hline 5 & 54 & 2.08 & 57.78 & 51 & 1.96 & 61.18 & 52 & 2.00 & 60.00 & 52.3 & 2.01 & 59.65 \\
\hline 6 & 49 & 1.88 & 63.67 & 55 & 2.12 & 52.73 & 48 & 1.85 & 65.00 & 50.6 & 1.95 & 61.80 \\
\hline 7 & 57 & 2.19 & 54.74 & 50 & 1.92 & 62.40 & 55 & 2.12 & 56.73 & 54.0 & 2.08 & 57.95 \\
\hline Average & & & & & & & & & & 51.6 & 1.98 & 60.78 \\
\hline
\end{tabular}

